\title{
ARTICLE
}

\section{Depression in schizophrenia and early psychosis: implications for assessment and treatment}

\author{
Rachel Upthegrove
}

Rachel Upthegrove is a consultant psychiatrist for South Birmingham Early Intervention Service and an honorary clinical senior lecturer at the University of Birmingham. Dr Upthegrove has been a member of the Royal College of Psychiatrists since 1999 and became a College tutor in 2007. Her research interests include insight in schizophrenia and affective disturbance in first-episode psychosis.

Correspondence Dr Rachel Upthegrove, Birmingham and Solihull Mental Health Trust, Early Intervention Service, 1 Miller Street, Aston, Birmingham B6 4NF, UK. Email: rachel.upthegrove@bsmht. nhs.uk

\begin{abstract}
SUMMARY
Depression in schizophrenia has been a neglected field for some time, but much evidence exists as to the common occurrence of affective disturbance in so-called non-affective psychosis. Depression is related to poor outcome in terms of functional recovery, relapse rate and suicide, and there is increasing evidence that it also precedes the onset of first-episode psychosis for many individuals. This article reviews recent steps forward in the evidence base for depression in schizophrenia in terms of prevalence, importance, assessment and treatment implications, with the aim of providing useful information for practising psychiatrists.
\end{abstract}

\section{DECLARATION OF INTEREST}

None.

The focus of European psychiatry and, since the publication of DSM-III and DSM-IV (American Psychiatric Association 1980, 1994), that of the rest of the world on positive and first-rank symptoms of schizophrenia to clarify and unify our diagnostic skills was an important step forward for psychiatry in the mid- to late-20th century. However, as increasing numbers of authors have proclaimed, psychiatrists have been in danger of throwing the baby out with the bath water by placing so little emphasis on the affective symptoms commonly experienced by people with schizophrenia. As illustrated by the case vignette below, difficulties arise in untangling the psychopathology when clear depressive symptoms are present. The approach to date has been to put less emphasis on the depressive symptoms, which are 'trumped' in diagnostic terms by the positive and first-rank symptoms. In the 1980s, Crow (1986) began the modern discontented chorus, opting for a unitary concept of psychosis, and subsequent investigation of classification systems by the UK700 group (van Os 2000) found little evidence of positive predictive value of diagnostic groups for positive, negative, depressive, manic and disorganisation symptoms. The conclusion is that the current distinction appears to obscure the natural overlap between symptom dimensions and that there is no real point of rarity between affective and non-affective psychotic diagnoses (van Os 2000; Murray 2005). In the following fictitious case vignette, depression is present in the prodrome and post-psychotic phases, yet with the absence of clear manic or mood congruent symptoms in the acute phase, a diagnosis of bipolar disorder would be inaccurate.

\section{Case vignette}

M.J. experienced her first psychotic episode at the age of 25. She remembers feeling low and anxious for 8 months before becoming more convinced that her personal safety was in jeopardy. She would watch passers-by in the street intently, monitoring their movements for signs of threat. She became increasingly isolated. Over a period of 8 weeks these feelings intensified to formed persecutory delusions regarding work colleagues, accompanied by poor sleep, agitation and external auditory hallucinations. She heard voices that made derogatory comments about her and her family but also commented on her actions. M.J. was admitted under Section 2 of the Mental Health Act 1983 to a busy acute adult ward. Here, her behaviour was bizarre, her affect incongruous, her self-care extremely poor and she was noted to be responding to unseen stimuli. M.J. was given intramuscular medication under restraint before accepting oral medication. She rapidly recovered and was discharged after 4 weeks on olanzapine $20 \mathrm{mg}$.

M.J. struggled to return to work and reported feeling increasingly low, with fully formed ideas of self-harm. She reported intrusive flashbacks to her initial stay in hospital and being restrained. She was shamed by having 'lost her mind' and felt she had no control over becoming ill again.

M.J. was commenced on fluoxetine $20 \mathrm{mg}$ while continuing with olanzapine at a low dose, and engaged in structured psychological therapy. She was initially reluctant to discuss her experiences in hospital or previous positive symptoms, and identified her main problems as a low sense of self-esteem and difficulty in trusting others. These remained the early targets of cognitive-behavioural therapy, while work with her keyworker focused on engagement, psychoeducation and relapse prevention. M.J. made a full recovery from both her first-episode psychosis and depression, and returned to full-time work some 9 months later. 
Bleuler (1911) first argued that problems of affect lie at the heart of schizophrenia and in keeping with this we now recognise that depression is extremely common in schizophrenia. Research has at last begun to refocus on the importance of affect in schizophrenia and early psychosis (Siris 2003; Birchwood 2005; Bentall 2006).

\section{Origin of depression in schizophrenia}

Many different models have been proposed to explain the link between depression and schizophrenia. As summarised in Table 1, depression has been described as an intrinsic part of the syndrome itself, as a side-effect of antipsychotic medication, as an expression of negative symptoms or as a psychological reaction to psychosis as a major life event. It has been hypothesised (Häfner 2005) that the depressive syndrome is a preformed reaction pattern associated with clear neurotransmitter dysfunction, progressing as the underlying brain dysfunction progresses: a Jacksonian hierarchical model whereby in the pathway to brain dysfunction severe enough to produce psychotic symptoms, the lesser neural networks will inevitably be disordered. In contrast, the psychological response conception has been modelled as a cognitive process. Birchwood et al (2005) found that over two-thirds of people who heard voices had moderate depression because of the experience and this was related not to the frequency or loudness of the voices, but to patients' appraisal (beliefs) of their voices' power and ability to harm or shame. Freeman et al (2001), in relation to the cognitive reaction to persecutors, found higher depression and distress to be associated with higher power and omnipotence of persecutors, but mitigated by patients' ability to avoid the threat ('safety behaviours'). Patients' appraisal of the diagnosis of psychosis and its impact on their social roles and status in terms of loss, shame and entrapment can also have predictive value in the development of post-psychotic depression (Iqbal 2000).

\section{The scale of the problem}

Medline and PsychLit searches using the search term 'depression AND schizophrenia' as major descriptors revealed over 577 hits since 2000 alone. Siris \& Bench (2003) reported on 40 published studies that documented depressive symptomatology in schizophrenia. Depression in schizophrenia is now accepted as common; however, the variance in rates of depression (Table 2) depends on whether the depressive syndrome or single symptoms are being reported, whether point or lifetime prevalence is being explored and what stage of schizophrenia is being studied.
TABLE 1

Proposed origins of depression in schizophrenia

\begin{tabular}{|ll|}
\hline Model & Comment \\
\hline Intrinsic to the illness itself & $\begin{array}{l}\text { A Jacksonian hierarchy: all 'lesser' neurobiological } \\
\text { abnormalities will be common when mental illness severe } \\
\text { enough to produce psychotic symptoms exists }\end{array}$ \\
\hline Medication effects & $\begin{array}{l}\text { For example: bradykinesia, loss of spontaneity, sedation, } \\
\text { akathisia }\end{array}$ \\
\hline $\begin{array}{l}\text { An expression of negative } \\
\text { symptoms }\end{array}$ & $\begin{array}{l}\text { The overlap of biological symptoms of depression with negative } \\
\text { symptoms (e.g. motor retardation) and symptoms of apathy }\end{array}$ \\
\hline Psychological response model & $\begin{array}{l}\text { Emphasis on the personal significance of psychosis and positive } \\
\text { symptoms. Appraisals of loss, shame and entrapment common }\end{array}$ \\
\hline
\end{tabular}

\section{Prevalence of depression in the prodrome}

The initial prodrome of psychosis is a potentially important period for early intervention (McGorry 2005). Individuals can be accurately recognised within the prodromal phase and interventions at this point can alleviate, delay or even prevent transition to a full psychotic episode (McGorry 2000; Yung 2003). Despite early ambiguity, it is now accepted that core symptoms commonly present in prodromal and high-risk groups (Parker 2006). What is striking, however, is the strong presence of depressive symptoms in prodromal clusters in both retrospective and prospective study designs (Yung 2003; Parker 2006; Rosen 2006). Yung et al (2003) found rates of depressive symptoms of $76 \%$ and identified high levels of emotional disturbance as being more predictive of transition to psychosis than subthreshold positive symptoms. McGorry and colleagues (2002) found that $45 \%$ of high-risk groups met criteria for DSM depressive disorder. Further, an der Heiden \& Häfner (2000) in their first-episode sample reported that $83 \%$ of their sample showed a depressed mood in the 2 weeks before first admission, with depressive symptoms appearing on average 52 weeks before first admission. Results from the Edinburgh High Risk Study (Cunningham Owens 2006) showed that anxiety and depression were very common in the high-risk sample long before those individuals developed frank psychotic symptom features. These affective symptoms were also significantly worse in those who subsequently experienced firstepisode schizophrenia.

\section{TABLE 2 Prevalence of depression by phase of illness}

\begin{tabular}{ll|}
\hline Phase of illness & Reported prevalence, \% \\
\hline Prodrome & $45-83^{\mathrm{a}}$ \\
\hline Acute & $29-75^{\mathrm{b}}$ \\
\hline Post-psychotic & $5-54^{\mathrm{c}}$ \\
\hline \\
$\begin{array}{l}\text { a. McGorry (2002); Yung (2003). } \\
\text { b. Koreen (1993). } \\
\text { c. Birchwood (2000); Siris (2003). }\end{array}$
\end{tabular}


Within established schizophrenia, the presence of depression outside the acute episode can represent post-psychotic depression or herald a prodrome to relapse. What remains to be investigated is whether targeted therapy, in subsequent episodes, can alleviate or prevent relapse.

\section{Prevalence of depression in acute psychosis}

This area has attracted relatively little research, yet there is evidence that depression may be more evident in the acute phase of illness. Figures from the few studies that have investigated the acute phase show rates from 45 to $75 \%$ (Koreen 1993). Post-psychotic depression may largely revolve around how individuals perceive the implications of psychosis for their own identity. Similarly, one might expect the psychological reaction to current, ongoing positive symptoms in the acute episode to be important. Tapp et al (2001) demonstrated in established cases of schizophrenia that depression accompanied the acute episode in $29 \%$ of patients and this depression largely resolved with treatment of positive symptoms. The area of depression in the acute phase has begun to attract research interest (Birchwood 2005).

\section{Prevalence of post-psychotic depression}

In psychosis, the depressive syndrome that has been longest and most widely recognised is postpsychotic depression. The ICD-10 definition of post-psychotic depression (F20.4) requires that some schizophrenic symptoms, either positive or negative, must still be present but that they no longer dominate the clinical picture. If schizophrenic symptoms are no longer present, a depressive episode should be diagnosed (F32). If schizophrenic symptoms are still florid and prominent, the diagnosis should remain that of the appropriate schizophrenic subtype (F20.0-F20.3) (World Health Organization 1992: pp. 93, 94).

These criteria make many of the assumptions outlined at the start of this article - that the predominance of schizophrenia symptoms often precludes the diagnosis of a concurrent depressive syndrome.

Studies show significant rates of depression (ranging from 5 to $40 \%$ ) in chronic schizophrenia (Siris 2003). Zisook (2006) focused on older patients, finding a $20 \%$ point prevalence of severe depression (defined as a Hamilton Rating Scale for Depression score $>17$ ) and concluding that depressive symptoms are common in this age group.

In their detailed study of the ontology of postpsychotic depression, Birchwood et al (2000) identified depression in $70 \%$ of their sample at onset of psychosis (in an acute phase): of these,
54\% developed post-psychotic depression. Four distinct course types were identified:

- depressed at onset, improvement, return of depression

- no depression at onset; depressed at one or more follow-up points

- depressed at onset; no depression throughout the follow-up period

- no depression at onset or throughout the followup period.

When investigating psychological models of post-psychotic depression, Birchwood et al (2005) and Iqbal et al (2000) demonstrated that those who go on to develop depression reported greater loss, humiliation and entrapment arising from their psychosis, and were more likely to attribute the cause of the psychosis to themselves. During post-psychotic depression there was a lowering of self-esteem and hardening of these negative appraisals.

\section{Why is depression important?}

\section{Risk of suicide}

Depression in schizophrenia is important, not least because it is significantly associated with suicide (Verdoux 2001; Crumlish 2005). Completed suicide remains frequent in psychotic illness, with an estimated $4.9-10 \%$ of patients with schizophrenia taking their own lives (Hawton 2005). The rate of suicide also appears to be increasing, or at least not significantly reducing (Healy 2006). In the International Suicide Prevention Trial, suicidal behaviour in people with psychotic disorders was described as 'a seriously under-treated lifethreatening condition' (Alphs 2004).

The early phase of illness is a particularly highrisk period. Mortensen $\&$ Juel (1993) followed up 9156 patients after their first admission with schizophrenia and found that suicide accounted for $50 \%$ of deaths in men and 35\% of deaths in women. Suicide risk was particularly high in the first year of follow-up. In addition, the OPUS study (Nordentoft 2002), which focused on suicidal behaviour in first-episode psychosis in The Netherlands, found female gender, hopelessness, hallucinations and suicide attempts at baseline to be predictive of future suicide attempts.

In summary, we need to be on high alert for the combination of depression and self-harm attempts in the early course of schizophrenia.

\section{Additional effects on outcome}

In addition to suicide risk, depression is linked to poor outcome in many other spheres. Relapse is more frequent and the total duration of in-patient 
treatment is longer in those with significant depression (an der Heiden 2005). Sands \& Harrow (1999) report depression to be significantly associated with unemployment at 4.5- and 7-year followup. Delahanty et al (2001) explored differences in depression according to ethnicity and reported that although depression is seven times higher in White people than in African Americans, indicating the possibility of underdiagnosis in the latter group, depression across all groups had a significant association with reduced quality of life.

\section{Assessment of depression in schizophrenia}

It is important to recognise that although depression in the prodrome of first-episode psychosis has some predictive validity for depression in the future (an der Heiden 2000), depression can also occur for the first time in the acute and post-psychotic phases (Iqbal 2000; Birchwood 2005). This area warrants further exploration to fully understand the patterns of depression through the course of schizophrenia, but in clinical practice doctors should be alert to the fact that depression can arise in any stage of the illness.

\section{Assessment instruments}

\section{The PANSS}

Studies of depression in schizophrenia have used a variety of assessment tools, some of which have useful clinical applications and can help untangle depressive syndrome from negative symptoms or medication side-effects. For example, the Positive and Negative Syndrome Scale (PANSS) (Kay 1987) is a valid, reliable and internationally recognised measure of the severity of positive and negative symptoms. It is divided into three subsections positive, negative and general psychopathology with depression scored within the last category. Depression is assessed with detailed questions on sadness, discouragement, pessimism and helplessness separate from negative psychopathology.

\section{The BDI and HRSD}

Also in frequent use and well known to most in a general setting are the Beck Depression Inventory (BDI) (Beck 1961) and the Hamilton Rating Scale for Depression (HRSD) (Hamilton 1960). Although the BDI is widely used, items such as psychomotor retardation, lack of energy, somatic preoccupation and 'an expectation of punishment' may overlap with negative symptoms and persecutory ideas common in schizophrenia. In addition, the selfreport construct makes its use difficult in acute psychosis. The HRSD is one of the most widely used instruments for measuring outcome in mood disorders and offers high validity and reliability in the assessment of depression. It contains items that assess somatic symptoms, insomnia, working capacity and interest, together with mood, guilt, psychomotor retardation and agitation. Similar to the BDI, the HRSD is not designed specifically for use in schizophrenia and there are clear areas of overlap.

\section{The CDSS}

The Calgary Depression Scale for Schizophrenia (CDSS) (Addington 1993) is a structured interview specifically designed for the assessment of depression in schizophrenia (www.ucalgary.ca/ cdss). The scale, which comprises eight structured questions and one interviewer observation, measures depression separately from negative and extrapyramidal symptoms. A score of 7 or more will have $82 \%$ specificity and $85 \%$ sensitivity to predict a moderate or severe depressive episode (Addington 1993).

\section{Comparative evaluation of instruments}

There have been relatively few comparative studies of assessment measures for depression in schizophrenia. Looking at the diagnostic validity of the four commonly used assessment scales mentioned above, Kim et al (2006) concluded that all had favourable sensitivity and specificity, but that validity was significantly greater for the CDSS. Comparative studies such as that by Kim et al have all advocated the use of the CDSS, as it is the only measure specifically designed for use in schizophrenia. The CDSS is unique in its ability to distinguish between depression, negative symptoms and extrapyramidal symptoms and, when compared with the HRSD and PANSS, is the most suitable measure of depression in schizophrenia. The combined use of the PANSS and CDSS is common in psychosis-specific clinical services. The CDSS is a good adjunct to clinical assessment when trying to untangle depression from negative symptoms or adverse effects of medication. I would advocate this as a clinically useful tool for all patients with schizophrenia.

(Key points 1.)

\section{KEY POINTS 1 Key assessment tools currently used for depression in psychosis}

- Calgary Depression Scale for Schizophrenia (Addington 1993)

- Positive and Negative Syndrome Scale (Kay 1987)

- Beck Depression Inventory (Beck 1961)

- Hamilton Rating Scale for Depression (Hamilton 1960) 


\section{Implications for treatment}

In 1967, Bowers \& Astrachan were impressed by the 'appearance and persistence of depression' in acute psychosis and commented that, after the first 4-6 weeks of treatment with phenothiazines, depression becomes the target of therapy for many patients. Thus, despite the preclusion of depressive symptoms from the classification system for schizophrenia and non-affective psychosis, pragmatic treatment has long acknowledged the need for this focus.

\section{Pharmacological treatment}

\section{Choice of antipsychotic}

In a description of 'depression in schizophrenia in the age of atypical antipsychotics', Siris (2000) proposed, in line with psychiatrists' optimism at the time, that schizophrenia treated with an atypical would have a different outcome and less depression. There is some merit in this, in that the increased extrapyramidal symptoms, akinesia and akathisia with first-generation antipsychotics may contribute to what is perceived as depression or other overlapping diagnoses such as anxiety and posttraumatic stress disorder. The atypicals offer an alternative to this. However, the hope that atypicals would reduce rates of depression by a direct influence on a more positive outcome and quality of life has not come to fruition (Swartz 2007). There is some evidence, however, that specific atypicals may have a more direct preventive effect on the development of depressive symptoms (Siris 2000).

Effective management of positive symptoms, taking keen account of the balance of side-effects, is a prerequisite in the treatment of depression in schizophrenia. It may be useful to consider antipsychotic dose reduction, if this is possible, and to treat extrapyramidal symptoms with an appropriate anti-Parkinsonian medication. Anti-Parkinsonian drugs may have their own antidepressant action; however, caution is necessary when using them with clozapine, owing to the combined anticholinergic effect. If a typical antipsychotic is being used, then substitution with an atypical should be considered. In keeping with National Institute for Health and Clinical Excellence (2002) guidelines, most people with first-episode schizophrenia in the UK are now treated with an atypical agent. However, the emergence of depression in a patient established on a typical antipsychotic should prompt consideration of changing to an atypical.

\section{Antidepressant medication}

Recent studies have focused on whether treatment with antidepressant medication during the schizophrenia can limit or prevent the onset of psychosis. Cornblatt et al (2007) demonstrated that a number of adolescents meeting criteria for prodromal schizophrenia were successfully treated with antidepressants, and advocate this as a first step in patients with prodromal symptoms presenting with high levels of depression. In patients who have experienced a first episode of psychosis, depressive symptoms may herald a damaging relapse; however, precise data on the predictive significance of depression in this instance are scarce. Given the association with poor outcome already discussed, the clinician should be alert to the significance of depression, carrying out more frequent monitoring and active treatment if symptoms persist.

In the acute phase, the incidence of depressive symptoms has been shown to reduce with active treatment of positive symptoms alone (Iqbal 2000). If this is not the case, then early treatment with antidepressant medication should be considered. Many authors advocate treatment with antidepressants only when the full depressive syndrome is apparent (Siris 2000). However, this would not reflect current practice in the management of major affective disorder, where the importance of effective management of residual symptoms is increasingly recognised (Fava 1999; Karp 2004).

A word of warning: many antidepressants have the potential to increase plasma concentrations of antipsychotic medication; for example, fluoxetine can increase clozapine concentrations by $50 \%$ (Lund 2001). Thus, there should be caution when combining antidepressants with higher doses of antipsychotics: increased monitoring such as is appropriate when exceeding British National Formulary limits might be necessary, as would the monitoring of plasma concentrations of the antipsychotic.

An international survey of antidepressant prescribing in schizophrenia (Addington 2002) demonstrated considerable international awareness of the prevalence and consequences of depression in schizophrenia. However, despite adjunctive use of antidepressants by many psychiatrists, a third of respondents indicated that they rarely or never prescribe antidepressants in combination with antipsychotic medication. Thus, in clinical practice the case for treatment with antidepressant medication is far from universally accepted. Indeed, a Cochrane review (Whitehead 2003) found only weak evidence to support the benefits of antidepressants in schizophrenia (but no evidence that antidepressants led to deterioration in psychotic symptoms). However, this looked only at a small number $(n=11)$ of relatively old studies, all randomising fewer than 30 patients, all of whom were on typical antipsychotics. 
To improve clarity on the benefits of antidepressant medication, larger randomised controlled trials are needed. Prior even to these, greater understanding must be acquired of the specific psychopathology and course of depression and during which phase of illness (prodrome, acute or post-psychotic) it occurs. Given the potential importance of depression in terms of relapse prevention, quality of life and suicide prevention, more focus within schizophrenia research should be directed here. Meanwhile, treatment should always include detailed assessment and careful weighting of risks and benefits, including risks to the patient of relapse and suicide.

\section{Psychological treatment}

Cognitive-behavioural therapy

Cognitive-behavioural therapy (CBT) is clearly efficacious in the treatment of positive symptoms and in reducing resistance to treatment in schizophrenia (Wykes 2007). Yet overall this effective intervention remains greatly under-used in clinical practice. A Cochrane review of CBT in schizophrenia (Jones 2004) cited the lack of proper randomised controlled trials and highlighted the considerable variability in the findings of the various studies examined. In reality there is insufficient knowledge of effective components and target areas within therapy and, in contrast to physical treatments, the efficacy of CBT has been studied before any real understanding or agreement of its efficacious component. Cognitivebehavioural therapy has, in effect, been evaluated as an antipsychotic, with most studies focusing on its usefulness for positive, or occasionally negative, symptoms.

Cognitive-behavioural therapy specifically for depression in schizophrenia has not been studied extensively. In their meta-analysis of CBT in psychosis, Wykes et al (2007) identified 15 studies that included measures of mood in the outcome of treatment, with a total sample size of 953 patients, and reported an overall improvement in mood with treatment significant at the 5\% level. In the only review of CBT for depression in psychosis, Birchwood et al (2004) highlighted nine randomised controlled trials that measured depression as an outcome measure for the effectiveness of CBT. None was specifically designed for this outcome, all measuring depression as a secondary outcome. This illustrates the need for more targeted phasespecific studies on individual outcomes and shows that CBT is not a panacea for psychosis and all its components.

Morrison et al (2004) advocate cognitive treatment for individuals in high-risk prodromal groups; the compelling rationale for this is the predominance of mood-related symptoms. Garety and colleagues (2001) place even more importance on the psychological treatment of prodromal depression, stating that the transition to frank psychosis is in fact driven by this depression. As rehearsed above, often it is the affective disturbance in high-risk groups that best predicts future psychotic illness (Cunningham Owens 2006). Birchwood \& Trower (2006) also derive clear treatment implications from their studies of post-psychotic depression and emphasise the importance of focusing on patients' appraisals of the threat posed by the diagnosis, by persecutors and by perceived social shame. They believe that therapy aimed at promoting adaptation to psychosis must focus on the resolution of appraisals of shame and entrapment, in a coherent and theoretically driven way. 'Scattershot' psychological approaches such as psychoeducation and relapse prevention, often early targets in the first episode, will not clearly identify these issues. Early aims of psychological treatment should more explicitly target negative appraisals in order to make an impact on depression. There are clear links between regaining insight and post-psychotic depression (Crumlish 2005). Making efforts to increase insight through psychoeducation without dealing with accompanying negative appraisals and implications runs the risk of having a negative rather than positive effect on outcome.

It is now widely accepted that CBT is useful in the treatment of depression (National Institute for Health and Clinical Excellence 2004), and patients with schizophrenia who experience depression should not be denied access to an effective treatment because of clinicians' preconceived and outdated ideas of their suitability for psychological interventions.

\section{Broader psychological intervention}

Cognitive-behavioural therapy should not become the only psychological therapy: psychoeducation, family therapy and relapse-prevention strategies are also important. The need for such a variety of focused treatments early in the course of psychotic illness emphasises the need for designated skilled early intervention teams and highlights the dearth of treatments previously offered to those with such a severe and devastating mental illness.

(Key points 2.)

\section{Conclusions}

Depression in all phases of schizophrenia and first-episode psychosis is common and related to poor outcome, from transition to active psychosis, relapse and suicide. 


\begin{tabular}{|llll|}
\multicolumn{4}{l}{ MCO answers } \\
1 & 2 & 3 & 4 \\
af & at & af & at \\
bf & bf & bt & bf \\
cf & cf & cf & cf \\
dt & df & df & df \\
ef & ef & ef & ef
\end{tabular}

KEY POINTS 2 Management of depression in psychosis

- Consider prescribing antidepressant medication

- Consider reducing the antipsychotic dose to minimise side-effects

- Treat extrapyramidal symptoms with antiParkinsonian medication

- Be aware of the combined anticholinergic effect of anti-Parkinsonians prescribed with clozapine

- Consider change of antipsychotic

- Be aware that antidepressants may increase plasma concentrations of antipsychotics

- Offer psychological interventions as a matter of course: CBT, psychoeducation, family therapy and relapse-prevention strategies

Although the underlying causes of depression in schizophrenia remain important areas of exploration, currently there are no clear answers. Certainly there appear to be different mechanisms involved in different phases of illness. How far this informs our debate on classification of psychotic illness is again an important point. However, this uncertainty should not detract from our knowledge that depression can be accurately distinguished from medication effects and negative symptoms, and that the treatment of this depression is important. The use of structured assessment measures can help here. In addition, to achieve a full functional recovery, prevent relapse and negative outcomes for patients with schizophrenia, depression should be a clear focus of both pharmacological and psychological treatment.

\section{References}

Addington D, Addington J, Maticka-Tyndale E (1993) Assessing depression in schizophrenia: the Calgary Depression Scale. British Journal of Psychiatry; 162 (suppl 22): 39-44.

Addington DD, Azorin JM, Falloon IR, et al (2002) Clinical issues related to depression in schizophrenia: an international survey of psychiatrists. Acta Psychiatrica Scandinavica; 105: 189-95.

*Alphs L, Anand R, Islam MZ, et al (2004) The international suicide prevention trial interSePT: rationale and design of a trial comparing the relative ability of clozapine and olanzapine to reduce suicidal behavior in schizophrenia and schizoaffective patients. Schizophrenia Bulletin; 30: $577-86$

American Psychiatric Association (1980) Diagnostic and Statistical Manual of Mental Disorders (3rd edn) (DSM-III). APA.

American Psychiatric Association (1994) Diagnostic and Statistical Manual of Mental Disorders (4th edn) (DSM-IV). APA.

an der Heiden W, Häfner $H$ (2000) The epidemiology of onset and course of schizophrenia. European Archives of Psychiatry and Clinical Neuroscience: 250: 292-303

an der Heiden W, Könnecke R, Maurer K (2005) Depression in the longterm course of schizophrenia. European Archives of Psychiatry and Clinical Neuroscience; 255: 174-84.

Beck AT, Ward CH, Mendleson M, et al (1961) An inventory for measuring depression. Archives of General Psychiatry; 4: 561-71.
*Bentall R (2006) Madness explained: why we must reject the Kraepelinian paradigm and replace it with a 'complaint-orientated' approach to understanding mental illness. Medical Hypotheses; 66: 220-33.

Birchwood M, Iqbal Z, Chadwick P, et al (2000) Cognitive approach to depression and suicidal thinking in psychosis. I. Ontogeny of postpsychotic depression. British Journal of Psychiatry; 177: 516-28.

Birchwood M, Iqbal Z, Jackson C, et al (2004) Cognitive therapy and emotional dysfunction in early psychosis. In Psychological Interventions in Early Psychosis: A Treatment Handbook (eds JFM Gleeson, PD McGorry): 209-28. John Wiley \& Sons.

Birchwood M, Iqbal Z, Upthegrove R (2005) Psychological pathways to depression in schizophrenia: studies in acute psychosis, post psychotic depression and auditory hallucinations. European Archives of Psychiatry and Clinical Neuroscience; 255: 202-12.

*Birchwood M, Trower P (2006) The future of cognitive-behavioural therapy for psychosis: not a quasi-neuroleptic. British Journal of Psychiatry; 188: 107-8.

Bleuler E (1911) Dementia Praecox. The Group of Schizophrenias (trans J. Zinkin, 1950). International Universities Press.

Bowers Jr MB, Astrachan BM (1967) Depression in acute schizophrenic psychosis. American Journal of Psychiatry; 123: 976-9.

Cornblatt BA, Lencz T, Smith C, et al (2007) Can antidepressants be used to treat the schizophrenia prodrome? Results of a prospective, naturalistic treatment study of adolescents. Journal of Clinical Psychiatry; 68 : 546-57

Crow TJ (1986) The continuum of psychosis and its implication for the structure of the gene. British Journal of Psychiatry; 149: 419-29.

Crumlish N, Whitty P, Kamali M, et al (2005) Early insight predicts depression and attempted suicide after 4 years in first-episode schizophrenia and schizophreniform disorder. Acta Psychiatrica Scandinavica; 112: 449-55.

*Cunningham Owens DG, Johnstone EC (2006) Precursors and prodromata of schizophrenia: findings from the Edinburgh High Risk Study and their literature context. Psychological Medicine; 36: 1501-14.

Delahanty J, Ram R, Postrado L, et al (2001) Differences in rates of depression in schizophrenia by race. Schizophrenia Bulletin; 27: 29-38.

Fava G (1999) Subclinical symptoms in mood disorders: pathophysiological and therapeutic implications. Psychological Medicine; 29: 47-61.

*Freeman D, Garety PA, Kuipers E (2001) Persecutory delusions: developing the understanding of belief maintenance and emotional distress. Psychological Medicine; 31: 1293-306.

*Garety PA, Kuipers E, Fowler D, et al (2001) A cognitive model of the positive symptoms of psychosis. Psychological Medicine; 31: 189-95.

Hamilton M (1960) A rating scale for depression. Journal of Neurology, Neurosurgery and Psychiatry; 23: 56-62.

Häfner H, Maurer K, Günteret T, et al (2005) Schizophrenia and depression: challenging the paradigm of two separate diseases - a controlled study of schizophrenia, depression and healthy controls. Schizophrenia Research; 77: $11-24$

*Hawton K, Sutton L, Haw C, et al (2005) Schizophrenia and suicide: systematic review of risk factors. British Journal of Psychiatry; 187: 9-20.

Healy D, Harris M, Tranter R, et al (2006) Lifetime suicide rates in treated schizophrenia: 1875-1924 and 1994-1998 cohorts compared. British Journal of Psychiatry; 188: 223-8.

Iqbal Z, Birchwood M, Chadwick P, et al (2000) Cognitive approach to depression and suicidal thinking in psychosis. 2. Testing the validity of a social ranking model. British Journal of Psychiatry; 177: 522-8.

Jones C, Cormac I, Silveira da Mota Neto Jl, et al (2004) Cognitive behaviour therapy for schizophrenia. Cochrane Database of Systematic Reviews; issue 4: CD000524.

Karp JF, Buysse DJ, Houck PR, et al (2004) Relationship of variability in residual symptoms with recurrence of major depressive disorder during maintenance treatment. American Journal of Psychiatry: 161: 1877-84.

Kay SR, Fiszbein A, Opler LA (1987) The positive and negative syndrome scale (PANSS) for schizophrenia. Schizophrenia Bulletin; 13: 261-76. 
Kim SW, Kim SJ, Yoon BH, et al (2006) Diagnostic validity of assessment scales for depression in patients with schizophrenia. Psychiatry Research; 144: 57-63.

Koreen AR, Siris SG, Chakos M, et al (1993) Depression in first episode psychosis. American Journal of Psychiatry; 150: 1643-7.

Lund BC, Perry PJ, Miller DD, et al (2001) Treating depression in schizophrenia. American Journal of Psychiatry; 158: 1528-9.

McGorry PD, McKenzie D, Jackson HJD, et al (2000) Can we improve the diagnostic efficiency and predictive power of prodromal symptoms for schizophrenia? Schizophrenia Research; 42: 91-100.

McGorry PD, Yung AR, Phillips LJ, et al (2002) Randomized controlled trial of interventions designed to reduce the risk of progression to first-episode psychosis in a clinical sample with subthreshold symptoms. Archives of General Psychiatry; 59: 921-8.

McGorry PD (2005) Early intervention in psychotic disorders: beyond debate to solving problems. British Journal of Psychiatry; 187 (suppl 48): s108-10.

Morrison AP, French P, Walford L, et al (2004) Cognitive therapy for the prevention of psychosis in people at ultra-high risk: randomised controlled trial. British Journal of Psychiatry; 185: 291-7.

Mortensen PB, Juel K (1993) Mortality and the causes of death in first admitted schizophrenic patients. British Journal of Psychiatry; 163: 183-9.

Murray V, McKee I, Miller PM, et al (2005) Dimensions and classes of psychosis in a population cohort: a four- class, four-dimension model of schizophrenia and affective psychoses. Psychological Medicine; 35 : 499-510.

National Institute for Health and Clinical Excellence (2002) Schizophrenia: Core Interventions in the Treatment and Management of Schizophrenia in Primary and Secondary Care. NICE.

National Institute for Health and Clinical Excellence (2004) Depression: Management of Depression in Primary and Secondary Care. NICE.

*Nordentoft M, Jeppesen P, Abel M, et al (2002) OPUS study: suicidal behaviour, suicidal ideation and hopelessness among patients with firstepisode psychosis. One-year follow-up of a randomised controlled trial. British Journal of Psychiatry; 181 (suppl 43): s98-106.

*Parker S, Lewis S (2006) Identification of young people at risk of psychosis. Advances in Psychiatric Treatment; 12: 249-55.

Rosen J, Miller T, D'Andrea J, et al (2006) Comorbid diagnoses in patients meeting criteria for the schizophrenia prodrome. Schizophrenia Research; 85: $124-31$.
Sands JR, Harrow M (1999) Depression during the longitudinal course of schizophrenia. Schizophrenia Bulletin; 25: 157-71.

* Siris SG (2000) Depression in schizophrenia: perspective in the era of 'atypical' antipsychotic agents. American Journal of Psychiatry; 157: 1379-89.

Siris SG, Bench C (2003) Depression and schizophrenia. In Schizophrenia (2nd edn) (eds SR Hirsch, D Weinberger): 142-67. Wiley-Blackwell.

Swartz MS, Perkins DO, Stroup TS, et al (2007) Effects of antipsychotic medications on psychosocial functioning in patients with chronic schizophrenia: findings from the NIMH CATIE study. American Journal of Psychiatry; 164: 428

Tapp A, Kilzieh N, Wood AE, et al (2001) Depression in patients with schizophrenia during an acute psychotic episode. Comprehensive Psychiatry; 42: 314-8

van Os J, Gilvarry C, Bale R, et al (2000) Diagnostic value of the DSM and ICD categories of psychosis: an evidence-based approach. Social Psychiatry and Psychiatric Epidemiology: 35: 305-11.

Verdoux H, Liraud F, Gonzales B, et al (2001) Predictors and outcome characteristics associated with suicidal behavior in early psychosis: a twoyear follow-up of first-admitted subjects. Acta Psychiatrica Scandinavica; 103: $347-54$

Whitehead C, Moss S, Cardno A, et al (2003) Antidepressants for the treatment of depression in people with schizophrenia: a systematic review. Psychological Medicine; 33: 589-99.

World Health Organization (1992) The ICD-10 Classification of Mental and Behavioural Disorders: Clinical Descriptions and Diagnostic Guidelines. WHO.

Wykes T, Steel C, Everitt B, et al (2007) Cognitive behavior therapy for schizophrenia: effect sizes, clinical models, and methodological rigor. Schizophrenia Bulletin; 34: 523-37.

Yung AR, Phillips LJ, Yuen HP, et al (2003) Psychosis prediction: 12-month follow up of a high-risk ('prodromal') group. Schizophrenia Research; 60: 21-32.

Zisook S, Nyer M, Kasckow J, et al (2006) Depressive symptom patterns in patients with chronic schizophrenia and subsyndromal depression. Schizophrenia Research: 86: 226-33.

*Publications of particular interest for further reading.

\section{MCQs}

1 Depression in schizophrenia is:

a only seen in the post-psychotic phase

b not related to poor outcome

c always the result of medication side-effects

$d$ an important early target of treatment

e the result of negative symptoms.

\section{Treatment of depression in schizophrenia should include:}

a consideration of cognitive-behavioural therapy

b high-dose antipsychotic medication

c clozapine d psychoeducation

e behavioural family therapy.

3 Assessment of depression in schizophrenia:

a is impossible in the presence of acute symptoms

$\mathrm{b}$ is made more accurate by the use of assessment scales such as the CDSS

c is of little importance compared with positive and first-rank symptoms

d invariably overlaps with that of negative symptoms e should be done only in the post-psychotic phase.

4 The prevalence of depression in schizophrenia:

a varies depending on whether single symptoms or full syndrome is being reported

$\mathrm{b}$ is low in the prodrome of first episode

c is invariably highest in the post-psychotic phase $\mathrm{d}$ has been overemphasised in recent years $\mathrm{e}$ is readily recognised by most psychiatrists. 